\title{
Prognostic significance of PI3K/AKT/mTOR signaling pathway members in clear cell renal cell carcinoma
}

\author{
Demin Fan Equal first author, 1 , Qiang Liu ${ }^{\text {Equal first author, }{ }^{2}, \text { Fei Wu }{ }^{1} \text {, Na Liu }}{ }^{3}$, Hongyi Qu ${ }^{4}$, Yijiao Yuan ${ }^{4}$, Yong Li ${ }^{5}$, Huayu Gao $^{4}$, \\ Juntao Ge ${ }^{4}$, Yue Xu ${ }^{4}$, Hao Wang ${ }^{4}$, Qingyong Liu ${ }^{1}$, Zuohui Zhao ${ }^{\text {Corresp. } 4}$ \\ 1 Urology, The First Affiliated Hospital of Shandong First Medical University, Jinan, China \\ Laboratory of Microvascular Medicine, The First Affiliated Hospital of Shandong First Medical University, Jinan, China \\ 3 Hematology, Qilu Hospital of Shandong University, Jinan, China \\ 4 Department of Pediatric Surgery, The First Affiliated Hospital of Shandong First Medical University, Jinan, China \\ 5 Urology, Shandong Yuncheng County Chinese Medicine Hospital, Heze, China \\ Corresponding Author: Zuohui Zhao \\ Email address: zhaozuohui@sdhospital.com.cn
}

Background. Renal cell carcinoma (RCC) is a fatal disease, in which the PI3K/AKT/mTOR signaling pathway serves an important role in the tumorigenesis. Previous studies have reported the prognostic significance of PI3K/AKT/mTOR signaling pathway members in RCC; however, there is insufficient evidence to date to confirm this. Thus, the present study aimed to systematically investigate the prognostic roles of multiple PI3K/AKT/mTOR signaling proteins in clear cell RCC (CCRCC) using online large-scale databases.

Methods. The mRNA expression profiles of PI3K/AKT/mTOR signaling pathway proteins PTEN, PIK3CA, $P I K 3 C B, P I K 3 C D$, PIK3CG, AKT1, AKT2, AKT3 and mTOR were investigated using the Gene Expression Profiling Interactive Analysis (GEPIA) and Oncomine databases, and the protein expression levels of PI3K, AKT and mTOR were detected using western blotting (WB) analysis. In addition, the correlation between mRNA or protein expression levels and the prognostic significance was analyzed using Kaplan-Meier (KM) plotter $(n=530)$, the Human Protein Atlas (HPA; $n=528)$ and The Cancer Protein Atlas (TCPA; $n=445)$ databases.

Results. The GEPIA revealed that the mRNA expression of major PI3K/AKT/mTOR pathway members, including PTEN, PIK3CA, PIK3CB, AKT1, AKT2 and AKT3, were negatively correlated with CCRCC stages $(\mathrm{P}<0.05)$, though most of their mRNA and protein expression levels were notsignificantly different between cCRCC and normal tissues using GEPIA, Oncomine and WB analyses ( $>0.05)$. Meanwhile, using the K-M plotter and HPA prognostic analysis, it was found that the mRNA expression levels of the majority of the PI3K/AKT/mTOR signaling pathway members, including PTEN, PIK3CA, PIK3CB, PIK3CG, AKT3 and mTOR were positively correlated with overall survival (OS), whereas PIK3CD mRNA expression was negatively correlated with OS $(\mathrm{P}<0.05)$. Furthermore, TCPA prognostic analysis observed that several of the key molecules of the PI3K/AKT/mTOR signaling pathway [PTEN, p-AKT (S473) and p-mTOR (S2448)] were also positively correlated with OS in patients with CCRCC $(P<0.05)$. In conclusion, the present study suggested that several members of the PI3K/AKT/mTOR signaling pathway, especially PTEN, may be favorable prognostic factors in cCRCC, which indicated that the PI3K/AKT/mTOR signaling pathway may be implicated in CCRCC initiation and progression. 
1 Prognostic significance of PI3K/AKT/mTOR signaling pathway members in clear cell renal 2 cell carcinoma

3 Demin $\mathrm{Fan}^{1 *}$, Qiang $\mathrm{Liu}^{2 *}$, Fei Wu${ }^{1}, \mathrm{Na} \mathrm{Liu}^{3}$, Hongyi Qu ${ }^{4}$, Yijiao Yuan ${ }^{4}$, Yong $\mathrm{Li}^{5}$, Huayu $\mathrm{Gao}^{4}$,

4 Juntao $\mathrm{Ge}^{4}$, Yue $\mathrm{Xu}^{4}$, Hao Wang ${ }^{4}$, Qingyong Liu${ }^{1}$, Zuohui Zhao ${ }^{4 \#}$

$5 \quad{ }^{1}$ Urology, The First Affiliated Hospital of Shandong First Medical University, Jinan, Shandong,

6 China

$7 \quad{ }^{2}$ Laboratory of Microvascular Medicine, The First Affiliated Hospital of Shandong First Medical

8 University, Jinan, Shandong, China

$9{ }^{3}$ Hematology, Qilu Hospital of Shandong University, Jinan, Shandong, China

$10{ }^{4}$ Department of Pediatric Surgery, The First Affiliated Hospital of Shandong First Medical

11 University, Jinan, Shandong, China

$12{ }^{5}$ Urology, Shandong Yuncheng County Chinese Medicine Hospital, Heze, Shandong, China

$13 *$ These authors contributed equally to this manuscript.

15 \# Corresponding author: Zuohui Zhao

16 Jingshi Road, No. 16766, Jinan, Shandong, 250014, China.

17 Email: zhaozuohui@sdhospital.com.cn. 


\section{ABSTRACT}

Background. Renal cell carcinoma (RCC) is a fatal disease, in which the PI3K/AKT/mTOR signaling pathway serves an important role in the tumorigenesis. Previous studies have reported the prognostic significance of PI3K/AKT/mTOR signaling pathway members in RCC; however, there is insufficient evidence to date to confirm this. Thus, the present study aimed to systematically investigate the prognostic roles of multiple PI3K/AKT/mTOR signaling proteins in clear cell RCC (ccRCC) using online large-scale databases.

Methods. The mRNA expression profiles of PI3K/AKT/mTOR signaling pathway proteins PTEN, PIK3CA, PIK3CB, PIK3CD, PIK3CG, AKT1, AKT2, AKT3 and $m T O R$ were investigated using the Gene Expression Profiling Interactive Analysis (GEPIA) and Oncomine databases, and the protein expression levels of PI3K, AKT and mTOR were detected using western blotting (WB) analysis. In addition, the correlation between mRNA or protein expression levels and the prognostic significance was analyzed using Kaplan-Meier (K-M) plotter ( $n=530)$, the Human Protein Atlas (HPA; $n=528)$ and The Cancer Protein Atlas (TCPA; $=445)$ databases.

Results. The GEPIA revealed that the mRNA expression of major PI3K/AKT/mTOR pathway members, including PTEN, PIK3CA, PIK3CB, AKT1, AKT2 and AKT3, were negatively correlated with ccRCC stages $(\mathrm{P}<0.05)$, though most of their mRNA and protein expression levels were not significantly different between ccRCC and normal tissues using GEPIA, Oncomine and WB analyses $(\mathrm{P}>0.05)$. Meanwhile, using the K-M plotter and HPA prognostic analysis, it was found that the mRNA expression levels of the majority of the PI3K/AKT/mTOR signaling pathway members, including PTEN, PIK3CA, PIK3CB, PIK3CG, AKT3 and $m$ TOR were positively correlated with overall survival (OS), whereas $P I K 3 C D$ mRNA expression was negatively correlated with OS $(\mathrm{P}<0.05)$. Furthermore, TCPA prognostic analysis observed that several of the key molecules of the PI3K/AKT/mTOR signaling pathway [PTEN, $p$-AKT (S473) and $p-m T O R(S 2448)]$ were also positively correlated with OS in patients with ccRCC $(\mathrm{P}<0.05)$. In conclusion, the present study suggested that several members of the PI3K/AKT/mTOR 
45 signaling pathway, especially PTEN, may be favorable prognostic factors in ccRCC, which

46 indicated that the PI3K/AKT/mTOR signaling pathway may be implicated in ccRCC initiation 47 and progression. 


\section{INTRODUCTION}

49 Kidney cancer is a high-risk cancer that demonstrates a high mortality rate, accounting for an estimated 73,750 newly diagnosed cases and 14,830 cancer-related deaths in the United States in 2020 (Siegel et al. 2020). Clear cell renal cell carcinoma (ccRCC), the most common subtype of kidney cancer, accounts for the majority of kidney cancer-related deaths(Rini et al. 2009). Localized RCC is commonly treated using nephrectomy and exhibits good prognosis; however, due to the resistance to traditional chemotherapy and radiotherapy, advanced RCC is considered a fatal malignancy with a poor overall survival (OS)(Rini et al. 2009). With the advances in tumor molecular genetics, the treatment for advanced RCC, in particular for metastatic RCC (mRCC), has evolved from traditional cytotoxic therapy to molecular targeted therapy, for example, sunitinib and everolimus, which have significantly prolonged the survival time for patients with RCC(Han et al. 2017). However, the molecular targeted therapy for RCC remains limited due to the lack of molecular targets. Thus, there is an urgent requirement to further investigate the molecular mechanisms that drive RCC initiation and progression, which will help identify potential therapeutic targets and prognostic markers for RCC therapy.

Apart from the well-known von Hippel-Lindau (VHL) gene, genetic mutations in members of the PI3K/AKT/mTOR signaling pathway are also frequently observed in RCC, which promotes the hyperactivation of the PI3K/AKT/mTOR signaling cascade(Han et al. 2017). The $\mathrm{PI} 3 \mathrm{~K} / \mathrm{AKT} / \mathrm{mTOR}$ signaling pathway, which is inhibited by phosphatase and tensin homolog (PTEN), is transduced through three checkpoint proteins or protein complexes: PI3K, AKT and mTOR. PTEN, PI3K, AKT and mTOR participate in the regulation of multiple biological functions, including cell survival, metabolism and tumorigenesis under context-specific physiological and pathological conditions (Cargnello et al. 2015; Guo et al. 2015). PTEN is a tumor suppressor gene, which is frequently mutated in tumors (Hager et al. 2011). PI3K consists of four isoforms: PIK3CA, PIK3CB, PIK3CD and PIK3CG. The activation of PI3K phosphorylates phosphatidylinositol-4,5-bisphosphate (PIP2) to generate phosphatidylinositol3,4,5-trisphosphate (PIP3), which subsequently recruits AKT to the plasma membrane, where 
75 AKT is activated by 3-phosphoinositide-dependent protein kinase (PDK) phosphorylation on

76 Thr308. This process can be reversed by PTEN, a phosphatase that can transform PIP3 to

77 inactivated PIP2. mTOR is a $289 \mathrm{kDa}$ serine/threonine kinase that encompasses two functionally

78 distinct protein complexes: mTOR complex 1 (mTORC1) and mTORC2 (Guo et al. 2015; Zhang

79 et al. 2017). Activated AKT phosphorylates mTORC1 on Ser2,448 to activate its kinase activity,

80 which then leads to the activation of multiple anabolic biosynthetic pathways that control cell

81 proliferation (Zhang et al. 2017). PI3K/AKT/mTOR signaling pathway dysregulation is

82 frequently identified in patients with RCC (Han et al. 2017), and inhibitors of mTOR, including

83 everolimus and temsirolimus, have proven efficacious in mRCC (Escudier et al. 2014; Ghidini et

84 al. 2017). In fact, previous studies have reported the prognostic value of PI3K/AKT/mTOR

85 signaling pathway-related proteins; however, the majority of these studies were based on small-

86 scale specimens and the conclusions lacked consistency (Hager et al. 2009; Kim et al. 2017;

87 Kruck et al. 2010; Liontos et al. 2017; Merseburger et al. 2008; Rausch et al. 2019; Zhu et al.

88 2015). Thus, it is necessary to investigate the prognostic role of the PI3K/AKT/mTOR signaling

89 pathway using large-scale databases.

90

91

92

93

94

95

96

97

98

By taking advantage of next-generation sequencing and multi-omics, a large number of potential biomarkers and therapeutic targets have emerged in recent years. The Cancer Genome Atlas (TCGA)(Han et al. 2017), The Cancer Proteome Atlas (TCPA) (Li et al. 2013), as well as several other large-scale cancer genome tools, such as Gene Expression Profiling Interactive Analysis (GEPIA) (Tang et al. 2019), Kaplan-Meier (K-M) plotter (Sui et al. 2019) and the Human Protein Atlas (HPA) (Zhu et al. 2018), have been comprehensively used to investigate the molecular profiles of several types of cancer. Nevertheless, only a few potentially useful prognostic biomarkers have been reported for RCC to date; for example, through screening the TCGA database, Han et al. (Han et al. 2017) discovered that numerous protein biomarkers, such as fatty acid synthase (FASN) and AKT3 were prognostic factors for the survival of patients with ccRCC; and using the Oncomine and K-M plotter databases, our previous study reported that several mRNA biomarkers, such as FASN and ATP citrate lyase (ACLY), may be prognostic 
102 factors for ccRCC (Zhao et al. 2019). In the present study, the mRNA and some protein

103

104

105

106

107

108

109

110

111

112

113

114

115

116

117

118

119

120

121

122

123

124

125

126

127

expression profiles of $\mathrm{PI} 3 \mathrm{~K} / \mathrm{AKT} / \mathrm{mTOR}$ signaling pathway members were compared between ccRCC and normal kidney tissues using the GEPIA, Oncomine and western blotting (WB) analyses, and their prognostic significance in ccRCC was subsequently systematically analyzed using the K-M plotter, HPA and TCPA databases (Fig. 1). In addition, the correlation between these $\mathrm{PI} 3 \mathrm{~K} / \mathrm{AKT} / \mathrm{mTOR}$ signaling pathway members and specific clinicopathological parameters of cancer, such as cancer stage, was also explored. In conclusion, the present study provided a comprehensive overview of the OS biomarkers found in the PI3K/AKT/mTOR signaling pathway, which highlighted the potential role of this pathway in ccRCC progression and targeted therapy.

\section{MATERIALS \& METHODS}

\section{GEPIA analysis}

The GEPIA server (http://gepia2.cancer-pku.cn/), which was based on the UCSC Xena project, was used to analyze the mRNA expression status of the following nine members of the $\mathrm{PI} 3 \mathrm{~K} / \mathrm{AKT} / \mathrm{mTOR}$ signaling cascade in ccRCC, as described previously (Tang et al. 2019): PTEN, PI3K (including PIK3CA, PIK3CB, PIK3CD and PIK3CG), AKT (including AKT1, AKT2 and $A K T 3$ ) and $m$ TOR. Briefly, the mRNA expression data of $523 \mathrm{ccRCC}$ and 100 normal kidney specimens from TCGA and Genotype-Tissue Expression (GTEx) were loaded into the server to analyze the differentially expressed genes between the two groups. The RNA-seq results were reported as the number of transcripts per million (TPM). The following filter indexes were set: expression mode was expression DIY, dataset was ccRCC, log-scale was log2 $(\mathrm{TPM}+1)$, and P-value cut-off was 0.05 . Then the mRNA expression parameters, including sample size and statistical box plot were displayed. For consistency analysis, the mRNA expression levels of the nine genes in $523 \mathrm{ccRCC}$ and 72 matched normal specimens from TCGA, and their correlationship between the genes and the pathological stages in $523 \mathrm{ccRCC}$ 
128

129

130

131

132

133

134

135

136

137

138

139

140

141

142

143

144

145

146

147

148

149

150

151

152

specimens were also analyzed.

\section{Oncomine analysis}

The Oncomine database (https://www.oncomine.org) was used to validate the mRNA expression levels of the PI3K/AKT/mTOR pathway members, as previously described (Zhao et al. 2018). After indexes were set: differential analysis (cancer versus normal tissue), cancer type (kidney cancer), and data type (mRNA), their differential expression parameters of the individual genes, such as sample size, P-value, fold changes and statistical box plot, were dispalyed.

\section{WB analysis}

All the patients signed the written informed consents before enrollment, and the procedures were approved by the ethical committees of The First Affiliated Hospital of Shandong First Medical University (No. 2017-S007). And 3 cases of ccRCC tissues (including cancer and adjacent noncancerous), which were collected from 3 patients ( 2 males and 1 females, age from 54 to 65 , Fuhrman nuclear grading with 1 G2 + 2 G3, TNM staging with 2 T1 + 1 T2) (Edge \& Compton 2010), were used for WB analysis as described previously (Zhao et al. 2017). After incubated with the corresponding primary antibodies: anti-PI3K (1:1,000, rabbit, \#4249; Cell signaling Technology, Danvers, MA), anti-AKT (1:1,000, rabbit, \#4691; Cell signaling Technology, Danvers, MA), anti-mTOR (1:2,000, rabbit, ab2732; Abcam, Cambridge, MA), and anti- $\beta$-Actin (1:1,000, mouse, BM0627; BOSTER, Beijing, China), horseradish peroxidase (HRP) conjugated secondary antibodies (1:5,000, rabbit, SA00001-2 \& mouse, SA00001-1; Proteintech, Wuhan, China) were used to detect the proteins. Then the blots were visualized by enhanced chemiluminescence (Amersham Imager 600, GE Healthcare, Marlborough, MA). Protein ladder (MAN0011772, Thermo Scintific, Waltham, MA) was used to label the corresponding proteins, and Image $\mathrm{J}$ software was used for quantification.

\section{K-M plotter mRNA survival analysis}

The K-M plotter (www.kmplot.com), which contained the RNA-seq data from Gene Expression 
153 Omnibus (GEO), Cancer Biomedical Informatics Grid (caBIG) and TCGA, was used to analyze

154 the correlation between the mRNA expression levels of each PI3K/AKT/mTOR signaling

155 pathway member and the prognosis of patients with ccRCC $(\mathrm{n}=530)$, as described previously

156 (Sui et al. 2019). First, the filter indexes were set: the pathological type was ccRCC, and auto

157 select best cut-off as the default cut-off. Then the nine PI3K/AKT/mTOR signaling pathway

158 genes (PTEN, PIK3CA, PIK3CB, PIK3CD, PIK3CG, AKT1, AKT2, AKT3 and $m T O R$ ) were

159 loaded into the online server, and the K-M survival plots were used to identify their correlation

160 with the OS for discovery analysis. The hazard ratio (HR), with 95\% confidence intervals (CI),

161 and the log-rank P-value were calculated automatically on the webpage after $\leq 150$ months

162 follow-up. The relationship between the gene expression levels and clinicopathological

163 parameters, for example, sex, pathological stages and grades, of ccRCC, were also investigated.

\section{HPA mRNA survival analysis}

The HPA database (www.proteinatlas.org), which retrieved the RNA-seq data from the Genomic Data Commons (GDC) Data Portal, was used to validate the prognosis of PI3K/AKT/mTOR signaling pathway genes in patients with ccRCC (Zhu et al. 2018). In the public database, the filter indexes were set: the pathological type was ccRCC, best expression cut-off as the default cut-off, then all the nine aforementioned genes were loaded into the database and the OS from the TCGA was used for validation analysis $(n=528)$. The K-M plot and log-rank P-value were also calculated accordingly after $\leq 150$ months follow-up.

\section{TCPA protein survival analysis}

TCPA database (https://tcpaportal.org/tcpa/) was used to analyze the correlation between the $\mathrm{PI} 3 \mathrm{~K} / \mathrm{AKT} / \mathrm{mTOR}$ signaling pathway protein expression levels and the prognosis of patients with ccRCC (Li et al. 2013). Briefly, seven proteins, including PTEN, PIK3CA, AKT, mTOR and three proteins modified by phosphorylation, phosphorylated (p)-AKT (S473), p-AKT (T308) and p-mTOR (S2448), were evaluated using TCPA database $(\mathrm{n}=445)$ after $\leq 150$ months followup. The log-rank and univariate Cox P-values were calculated automatically. The relationship 
179 between the protein expression levels and the clinicopathological parameters, for instance, the 180 pathological stage and Fuhrman grade of ccRCC, were also investigated.

181

182

183

184

185

186

187

188

189

190

191

192

193

194

195

196

197

198

199

200

201

202

203

\section{Statistical analysis}

Statistical analysis was performed using SPSS 21.0 software (IBM Corp.). For GEPIA analysis, the mRNA expression levels of PI3K/AKT/mTOR signaling pathway members in ccRCC and normal tissues were analyzed using one-way ANOVA. For Oncomine and WB analyses, the $\mathrm{PI} 3 \mathrm{~K} / \mathrm{AKT} / \mathrm{mTOR}$ expression levels were analyzed using a two-tailed Student's t-test. For the KM plotter and HPA analyses, the correlation between the mRNA expression levels and OS was calculated using K-M curves and the log-rank test. For TCPA analysis, the correlation between protein expression levels and OS was calculated using the log-rank test and a univariate Cox Proportional Hazards regression model. $\mathrm{P}<0.05$ were considered to indicate a statistically significant difference.

\section{RESULTS}

\section{GEPIA, Oncomine and WB analyses of the expression levels of PI3K/AKT/mTOR} signaling pathway members between ccRCC and normal tissues

First, the mRNA expression profiles of the PI3K/AKT/mTOR signaling pathway members were analyzed using GEPIA as described previously (Tang et al. 2019). The RNA-Seq data from TCGA and GTEx were used to compare the expression levels of nine PI3K/AKT/mTOR signaling pathway members (PTEN, PIK3CA, PIK3CB, PIK3CD, PIK3CG, AKT1, AKT2, AKT3 and $m T O R$ ) between ccRCC and normal kidney tissues. The mean mRNA expression levels of these genes were not significantly different between the 523 ccRCC and 100 normal tissues (P>0.05; Fig. 2); however, both PIK3CD and PIK3CG displayed an upward trend while $m$ TOR displayed a decreasing trend, but not significant expression levels in patients with ccRCC $(\mathrm{P}>0.05)$. Similar results were obtained between $523 \mathrm{ccRCC}$ and 72 matched normal samples 
204 using GEPIA analysis (Table S1).

205 Regarding the association of mRNA expression with pathological stages of the ccRCC patients, 206 the detailed expression levels of the PI3K/AKT/mTOR members were further investigated in the 207523 patients using GEPIA. The violin plots showed their expression in each stage, which 208 illustrated the mRNA expression levels of six genes, i.e., $P T E N(\mathrm{P}=0.002), P I K 3 C A(\mathrm{P}<0.001)$, 209 PIK3CB $(\mathrm{P}=0.010), A K T 1(\mathrm{P}<0.001), A K T 2(\mathrm{P}<0.001)$ and AKT3 $(\mathrm{P}<0.001)$, were negatively 210 correlated with their stages (Fig. 3).

211 Then Oncomine database (U133A/B microarray, 10 ccRCC and 10 matched normal kidney 212 specimens) was used to validate their differential expression (Table S2), which demonstrated that 213 most of the PI3K/AKT/mTOR members were not significantly changed between ccRCC and 214 normal specimens ( $\mathrm{P}>0.05)$ except PTEN (1.77-fold increase in ccRCC, $\mathrm{P}<0.001)$, PIK3CA 215 (1.56-fold increase, $\mathrm{P}=0.010)$ and AKT1 (1.27-fold increase, $\mathrm{P}=0.005)$.

216 Finally, further WB analysis displayed that the protein expression levels of PI3K, AKT and 217 mTOR were not significantly changed between ccRCC and normal specimens $(\mathrm{P}=0.774$, $218 \mathrm{P}=0.585, \mathrm{P}=0.480$, respectively; Fig. 4), which also consistent with the mRNA expression of $219 \mathrm{PI} 3 \mathrm{~K} / \mathrm{AKT} / \mathrm{mTOR}$ signaling members.

K-M plotter and HPA prognostic analysis of the PI3K/AKT/mTOR signaling pathway members in ccRCC

222 For survival analysis, the public online resource K-M plotter was used to calculate the clinical 223 significance of individual genes compared with OS in 530 patients with ccRCC (Fig. 5; Table 1). 224 The results demonstrated that increased mRNA expression levels of PTEN [HR 95\% CI $=0.56$ 225 (0.41-0.75); $\mathrm{P}<0.001]$ and $m$ TOR $[\mathrm{HR} 95 \% \mathrm{CI}=0.54$ (0.38-0.78); $\mathrm{P}<0.001]$ were associated with 226 improved OS in patients with ccRCC. For the multiple $A K T$ genes, $A K T 3$ [HR 95\% CI $=0.44$ 227 (0.32-0.62); $\mathrm{P}<0.001]$ was observed to be a favorable factor; however $A K T 1$ [HR 95\% CI $=0.76$ 228 (0.56-1.02); $\mathrm{P}=0.068]$ and $A K T 2$ [HR 95\% CI $=1.34$ (0.99-1.82); $\mathrm{P}=0.055]$ were not. As for the 
229

230

231

232

233

234

235

236

237

238

239

240

241

242

243

244

245

246

247

248

249

250

251

252

253

254

255

$P I 3 K$ genes, the majority were identified as protective factors, including PIK3CA [HR 95\% CI $=0.52$ (0.38-0.70); $\mathrm{P}<0.001], P I K 3 C B[\mathrm{HR} 95 \% \mathrm{CI}=0.50(0.37-0.68) ; \mathrm{P}<0.001]$ and $P I K 3 C G$ [HR 95\% CI $=0.64$ (0.47-0.87); $\mathrm{P}=0.004]$, whereas $P I K 3 C D$ demonstrated the opposite effect [HR 95\% CI $=1.38$ (1.01-1.91); $\mathrm{P}=0.046]$. These results suggested that different members of $\mathrm{AKT}$ or PI3K may serve distinct roles in the prognosis of ccRCC.

In addition to investigating OS, cancer staging, sex and grading were further investigated to determine the correlation between mRNA expression levels and OS in ccRCC patients. According to the TNM staging system (Edge \& Compton 2010), the ccRCC samples were grouped into four stages and the correlation between mRNA expression levels and OS in different pathological stages of ccRCC patients was analyzed (Table 2). It was observed that PTEN, PIK3CA and PIK3CB were positively correlated with a favorable OS in stage I, III and IV patients with ccRCC [HR 95\% CI =0.46 (0.25-0.85) (stage I); 0.44 (0.25-0.79) (stage III); 0.51 (0.30-0.87) (stage IV) for PTEN; 0.48 (0.26-0.88) (stage I); 0.51 (0.29-0.89) (stage III); 0.61 (0.37-1.00) (stage IV) for PIK3CA; and 0.43 (0.23-0.81) (stage I); 0.40 (0.22-0.70) (stage III); 0.41 (0.25-0.67) (stage IV) for PIK3CB, respectively]. Furthermore, increased PIK3CG expression levels were correlated with an improved OS in stage I, II and III patients with ccRCC [HR 95\% CI =0.53 (0.28-1.00); 0.29 (0.08-1.00); 0.31 (0.17-0.54), respectively]. Similar results were achieved for AKT3 and mTOR; AKT3 was found to be positively correlated with a favorable prognosis in stage III and IV patients with ccRCC [HR 95\% CI $=0.45(0.25-0.81) ; 0.50$ (0.27-0.94), respectively] and the overexpression of $m T O R$ was correlated with an improved OS in stage I and II patients with ccRCC [HR 95\% CI =0.30 (0.12-0.77); 0.24 (0.06-0.86), respectively]. AKT1 was discovered to be a favorable factor in stage I [HR 95\% CI $=0.44(0.24$ 0.80)], whereas it found to be a poor factor in stage II and III patients with ccRCC [HR 95\% CI $=3.00(0.98-9.20) ; 2.15$ (1.14-4.07), respectively]. PIK3CD mRNA expression levels were associated with a favorable OS in stage III [HR 95\% CI $=0.55(0.31-0.96)]$, but a worse OS in stage IV $[\mathrm{HR} 95 \% \mathrm{CI}=2.42(1.25-4.68)]$ patients with ccRCC. AKT2 mRNA expression was not correlated with the tumor stages of patients with $\operatorname{ccRCC}(\mathrm{P}>0.05)$. These results suggested that 
256 the prognostic role of $\mathrm{PI} 3 \mathrm{~K} / \mathrm{AKT} / \mathrm{mTOR}$ signaling pathway members may be markedly 257 influenced by TNM stages.

258 Regarding the association of these genes with the sex of patients, the prognostic values of 259 individual genes were further investigated in patients with ccRCC. As illustrated in Table 3, 260 PTEN, PIK3CA, PIK3CB, PIK3CG and AKT3 were positively correlated with a favorable OS in 261 both females [HR 95\% CI =0.35 (0.21-0.59) for PTEN; $0.27(0.15-0.51)$ for PIK3CA; $0.45(0.27$ 262 0.76) for PIK3CB; 0.49 (0.30-0.82) for PIK3CG; and $0.32(0.20-0.54)$ for $A K T 3$, respectively] 263 and males [HR 95\% CI =0.53 (0.36-0.77) for PTEN; $0.57(0.39-0.82)$ for PIK3CA; $0.48(0.33$ 264 0.70) for $P I K 3 C B ; 0.68(0.47-0.99)$ for $P I K 3 C G$; and 0.48 (0.31-0.72) for $A K T 3$, respectively]. mTOR was positively correlated with an improved OS in female patients with ccRCC [HR 95\% $\mathrm{CI}=0.27(0.14-0.52)]$, whereas $A K T 2$ was positively correlated with poor OS in males [HR 95\% $\mathrm{CI}=1.71(1.15-2.55)]$. Moreover, $P I K 3 C D$ was positively correlated with a favorable OS in females [HR 95\% CI $=0.57$ (0.34-0.97)], but poor OS in male patients with ccRCC [HR 95\% CI $=1.81(1.22-2.68)]$. These results suggested that sex may affect the prognostic role of $\mathrm{PI} 3 \mathrm{~K} / \mathrm{AKT} / \mathrm{mTOR}$ signaling pathway members in patients with ccRCC.

Similarly, the prognostic value of the genes in different grades of ccRCC was also evaluated. As illustrated in Table S3, the OS of the nine genes in different grades were consistent with the 273 total OS.

274 For HPA validation analysis, the prognostic significance of the nine genes was examined, 275 which found that the majority of genes demonstrated identical prognosis (OS) following the 276 same follow-up period in another 528 patients with ccRCC (Fig. 6). Specifically, increased 277 expression levels of PTEN ( $\mathrm{P}<0.001)$, PIK3CA $(\mathrm{P}<0.001)$, PIK3CB $(\mathrm{P}<0.001)$, PIK3CG $278(\mathrm{P}=0.004), A K T 3(\mathrm{P}<0.001)$ and $m$ TOR $(\mathrm{P}=0.001)$ were correlated with a significantly improved 279 OS in 528 patients with ccRCC, whereas the overexpression of $P I K 3 C D$ mRNA $(\mathrm{P}=0.048)$ was 280 significantly correlated with poor OS. AKT1 mRNA expression levels demonstrated no significant correlation with the prognosis of patients with $\operatorname{ccRCC}(\mathrm{P}=0.082)$, with the K-M 
282 plotter results providing similar findings. In addition, HPA analysis identified $A K T 2$ as a hazard 283 factor $(\mathrm{P}=0.004)$, but it was not correlated with patients' prognosis $(\mathrm{P}=0.055)$, which was 284 assessed using K-M plotter analysis.

285 286

287 288

289

290

291

292

293

294

295

296

297

298

299

300

301

302

303

304

305

306

307

\section{TCPA prognostic analysis of the protein expression levels of PI3K/AKT/mTOR signaling} pathway members in ccRCC

Since the activity of the $\mathrm{PI} 3 \mathrm{~K} / \mathrm{AKT} / \mathrm{mTOR}$ signaling cascade is largely regulated through phosphorylation events, the prognostic roles of core executors of the PI3K/AKT/mTOR signaling pathway, with or without phosphorylation, were investigated in patients with ccRCC. For this purpose, 445 cases of ccRCC from TCPA database were used for the statistical analysis (Fig. 7; Table 4). It was discovered that the higher expression levels of $P T E N$ (log-rank $\mathrm{P}<0.001$; Cox $\mathrm{P}<0.001), p-A K T$ (S473; log-rank $\mathrm{P}=0.047$; Cox $\mathrm{P}=0.002)$ and $p$-mTOR (S2448; log-rank $\mathrm{P}=0.004$; Cox $\mathrm{P}<0.001$ ) were associated with an improved $\mathrm{OS}$ in patients with ccRCC, which is consistent with previous findings (Zhang et al. 2017). Although $A K T$ and $m T O R$ were not recognized as significant prognostic factors by K-M plot analysis (log-rank $\mathrm{P}=0.263$ for AKT; $\mathrm{P}=0.543$ for $\mathrm{mTOR}$ ), they were identified as favorable prognostic factors by univariate Cox Proportional Hazards model analysis ( $\mathrm{Cox} \mathrm{P}=0.003$ for $\mathrm{AKT}$; $\mathrm{P}=0.041$ for $\mathrm{mTOR}$ ). However, PIK3CA and p-AKT (T308) expression levels were not correlated with tumor prognosis in patients with ccRCC $(\mathrm{P}>0.05)$.

As tumor staging and grading could markedly influence the statistical results of prognosis, correlation analysis between protein expression levels and ccRCC stages or grades were further performed (Table 4). The expression levels of PTEN, AKT and $p-A K T$ (S473) were positively correlated with both tumor stages $(\mathrm{P}<0.001 ; \mathrm{P}=0.045$ and $\mathrm{P}<0.001$, respectively) and tumor grades ( $\mathrm{P}<0.001 ; \mathrm{P}<0.001$ and $\mathrm{P}<0.001$, respectively) in patients with ccRCC, which is consistent with previous studies (Han et al. 2017). However, no significant correlation was observed between PIK3CA, $m$ TOR and $p-m T O R$ (S2448) expression and tumor stages or grades of patients with ccRCC $(\mathrm{P}>0.05)$. 


\section{DISCUSSION}

310

311

312

313

314

315

316

317

318

319

320

321

322

323

324

325

326

327

328

329

330

331

332

333

334

ccRCC is the most prevalent kidney cancer; however, its molecular mechanism remains to be fully elucidated (Han et al. 2017). The PI3K/AKT/mTOR signaling pathway, which is a central regulator of cell survival and proliferation, is constitutively active in RCC and reportedly implicated in RCC pathogenesis and progression. In the present study, the mRNA expression levels of the aforementioned nine genes demonstrated no significant differences between ccRCC and normal tissues $(\mathrm{P}>0.05)$, suggesting that the activation of the $\mathrm{PI} 3 \mathrm{~K} / \mathrm{AKT} / \mathrm{mTOR}$ signaling pathway in patients with ccRCC may not be dependent on their transcriptional regulation. Although WB displayed an upward trend of AKT protein expression in ccRCC, more samples were needed to clarify this issue. When striatified by clinical stage of the 523 ccRCC cases using GEPIA, the mRNA expression levels of six genes, i.e., PTEN, PIK3CA, PIK3CB, AKT1, AKT2 and $A K T 3$, were negatively correlated with their stages $(\mathrm{P}<0.05)$. Further prognostic analysis using the K-M plotter and HPA demonstrated that the mRNA expression levels of the major PI3K/AKT/mTOR signaling pathway members (PTEN, PIK3CA, PIK3CB, PIK3CG, AKT3 and $m T O R$ ) were positively correlated with a favorable OS in patients with ccRCC, whereas $P I K 3 C D$ mRNA expression levels were positively correlated with a poor $\mathrm{OS}(\mathrm{P}<0.05)$. Accordingly, TCPA analysis demonstrated that the protein expression levels of PTEN, p-AKT (S473) and pmTOR (S2448) were correlated with an improved OS $(\mathrm{P}<0.05)$. The relationship between their mRNA or protein expression levels and several clinicopathological features, such as sex, pathological stage and Fuhrman grade, were also analyzed. The results demonstrated that the majority of the stratified prognosis was consistent with the overall prognosis, which confirmed the validity of our conclusion. In general, the prognosis between the mRNA and protein expression levels of members of the $\mathrm{PI} 3 \mathrm{~K} / \mathrm{AKT} / \mathrm{mTOR}$ signaling pathway was consistent, with the majority being recognized as favorable prognostic factors in patients with ccRCC. The mRNA expression levels of the six genes, i.e., PTEN, PIK3CA, PIK3CB, AKT1, AKT2 and AKT3, were negatively correlated with their stages, which was also consistent with their favorable 
335 prognosis in ccRCC. This suggested that the function of PI3K/AKT/mTOR in ccRCC was not

336

337

338

339

340

341

342

343

344

345

346

347

348

349

350

351

352

353

354

355

356

357

358

359

360

361

only dependent on the mRNA and protein expression levels, but also reliant on protein activation through phosphorylation, such as $p-A K T$ and $p$-mTOR. What's more, it also indicated that the activation of PI3K/AKT/mTOR was a complicated process, and further molecular biological, pathological and biochemical experiments were needed to support the prognosis of $\mathrm{PI} 3 \mathrm{~K} / \mathrm{AKT} / \mathrm{mTOR}$ pathway members in ccRCC. To the best of our knowledge, this was the first report studying the prognostic role of mRNA and protein expression levels of multiple $\mathrm{PI} 3 \mathrm{~K} / \mathrm{AKT} / \mathrm{mTOR}$ pathway members, such as PTEN, AKT1 and $m T O R$, in ccRCC

simultaneously. Our findings indicated that they may be potential prognostic markers for ccRCC, in addition to suggesting that $\mathrm{PI} 3 \mathrm{~K} / \mathrm{AKT} / \mathrm{mTOR}$ pathway signaling abnormalities may be involved in ccRCC tumorigenesis and progression.

The PI3K/AKT/mTOR signaling pathway serves an important role in ccRCC pathogenesis and progression, and is negatively regulated by the tumor suppressor PTEN. PTEN has been identified as one of the most commonly lost or mutated tumor suppressor genes in human cancers (Que et al. 2018; Tang et al. 2017); however, although PTEN has been thoroughly investigated in RCC, its prognostic role remains controversial. For example, Zhu et al. (Zhu et al. 2015 ) reported that PTEN protein expression levels were decreased in RCC, and PTEN could be used as a favorable prognostic (OS) marker, whereas Kim et al. (Kim et al. 2017) discovered that PTEN was not an independent prognostic marker of RCC. However, two meta-analyses indicated that PTEN was a favorable prognostic factor for patients with ccRCC (Que et al. 2018; Tang et al. 2017), which is consistent with our findings. In recent years, research has also focused on investigating the prognosis of other PI3K/AKT/mTOR signaling pathway proteins using immunohistochemistry analysis. For example, Merseburger et al. (Merseburger et al. 2008) demonstrated that $P I 3 K$ activation was inversely correlated with RCC patients' survival, and that low PTEN/high $p$-AKT expression levels were also associated with a decreased survival in 176 patients with RCC; Pantuck et al. (Pantuck et al. 2007) found that high expression levels of nuclear $p$-AKT (S473) were associated with favorable disease-specific survival (DSS) in 375

Peer] reviewing PDF | (2020:01:45296:2:0:NEW 5 May 2020) 
362 patients with RCC; and Hager et al. (Hager et al. 2009) reported that increased $p-A K T$ (S473)

363

364

365

366

367

368

369

370

371

372

373

374

375

376

377

378

379

380

381

382

383

384

385

386

387

388

expression levels were correlated with a poorer OS for 440 patients with RCC. In addition,

Kruck et al. (Kruck et al. 2010) investigated the expression levels of $m$ TOR and p-mTOR (S2448)

in $10 \mathrm{ccRCC}$ and normal kidney tissues, and found that p-mTOR (S2448), but not mTOR

expression levels, were increased in ccRCC. A subsequent study by the same authors

comprehensively investigated the differential expression levels of mTOR and p-mTOR in 342

primary and 90 metastatic ccRCC tissues, and revealed that high p-mTOR expression levels, but not mTOR levels, were associated with impaired OS (Rausch et al. 2019). Furthermore, Liontos

et al. (Liontos et al. 2017) reported that post-treatment, the combination of increased p-mTOR expression levels and low VEGF expression levels was negatively correlated with OS in 79

patients with mRCC who were refractory to first-line sunitinib treatment. However, collectively these studies failed to reach a consistent conclusion and the prognostic role of the

$\mathrm{PI} 3 \mathrm{~K} / \mathrm{AKT} / \mathrm{mTOR}$ signaling pathway in RCC remains controversial. The prognostic

inconsistency of the PI3K/AKT/mTOR signaling pathway in RCC may be due to the remarkable

heterogeneity of RCC specimens, the limited sample size and the divergence of

clinicopathological backgrounds.

Integrated bioinformatics analysis confirmed that the PI3K/AKT/mTOR pathway was activated in ccRCC, but the prognostic significance was not fully elucidated(Cancer Genome Atlas Research 2013; Chen et al. 2016; Han et al. 2017; Zhang et al. 2017). Through pan-cancer proteogenomic atlas analysis, Zhang et al. (Zhang et al. 2017)found that PTEN, p-AKT and pmTOR expression levels were all significantly correlated with improved outcomes in 32 major types of cancer, which was also observed in the present study. Similar results were reported by Han, who demonstrated that certain members of the PI3K/AKT/mTOR signaling pathway, such as PTEN protein expression, and PTEN and AKT3 mRNA expression were prognostic factors for ccRCC (Han et al. 2017). However, no clarification was provided on whether these were associated with a favorable or unfavorable prognosis (Han et al. 2017). In addition, in 446 ccRCC cases, integrative proteogenomic analysis revealed that some members of the

Peer) reviewing PDF | (2020:01:45296:2:0:NEW 5 May 2020) 
389 390

391

392

393

394

395

396

397

398

399

400

401

402

403

404

405

406

407

408

409

410

411

412

413

414

$\mathrm{PI} 3 \mathrm{~K} / \mathrm{AKT} / \mathrm{mTOR}$ signaling pathway, for example, PTEN and AKT mRNA expression levels, and PTEN protein expression levels, were recognized as favorable predictors for ccRCC (Cancer Genome Atlas Research 2013), which was also consistent with our findings in the present study. Meanwhile, through multilevel genomics-based taxonomy of 894 RCC samples, Chen et al. (Chen et al. 2016) demonstrated that the PI3K/AKT/mTOR signaling pathway could distinguish between RCC subtypes, such as clear cell, chromophobe and papillary RCC; however the prognostic value was not investigated. In the present study, comprehensive analysis of the prognostic significance of components of this pathway at the mRNA and protein level was performed in $\sim 500$ ccRCC tissues using the K-M plotter, HPA and TCPA databases; it was found that the majority of the mRNAs (such as PTEN and AKT3) and several proteins [such as PTEN and $p$-AKT (S473)] were favorable prognostic factors for ccRCC, which indicated that the $\mathrm{PI} 3 \mathrm{~K} / \mathrm{AKT} / \mathrm{mTOR}$ signaling pathway may be implicated in ccRCC progression, which may also provide a potential therapeutic target for patients with ccRCC. These findings are contradictory to the common knowledge that the PI3K/AKT/mTOR signaling pathway promotes the initiation and progression of RCC. However, mTOR signaling is necessary for multiple biological effects, including energy metabolism homeostasis, autophagy, cell survival and apoptosis. Thus, the prognostic roles of $\mathrm{PI} 3 \mathrm{~K} / \mathrm{AKT} / \mathrm{mTOR}$ signaling pathway members in patients with RCC may be different under specific physiological or pathological conditions, although this hypothesis requires to be further investigated.

\section{CONCLUSIONS}

The present study investigated the mRNA expression profiles of multiple $\mathrm{PI} 3 \mathrm{~K} / \mathrm{AKT} / \mathrm{mTOR}$ signaling pathway components, including PTEN, PIK3CA, PIK3CB, PIK3CD, PIK3CG, AKT1, $A K T 2, A K T 2$ and $m T O R$, in ccRCC and normal tissues using GEPIA and Oncomine analyses, and detected PI3K, AKT and mTOR protein expression using WB analysis. The prognostic significance of these genes and proteins in ccRCC patients was evaluated using K-M plotter, 
415 HPA and TCPA analysis, which suggested that several members of the PI3K/AKT/mTOR 416 signaling pathway, especially PTEN, may be favorable prognostic factors in ccRCC. To the best 417 of our knowledge, our study was the first to reveal the detailed OS of nine PI3K/AKT $/ \mathrm{mTOR}$ 418 signaling pathway members simultaneously. These results further suggested that the $419 \mathrm{PI} 3 \mathrm{~K} / \mathrm{AKT} / \mathrm{mTOR}$ signaling pathway may be implicated in ccRCC initiation and progression, 420 and that components could be used as potential therapeutic targets in ccRCC.

\section{Acknowledgements}

Not applicable.

\section{References}

Cancer Genome Atlas Research N. 2013. Comprehensive molecular characterization of clear cell renal cell carcinoma. Nature 499:43-49. 10.1038/nature12222

Cargnello M, Tcherkezian J, and Roux PP. 2015. The expanding role of mTOR in cancer cell growth and proliferation. Mutagenesis 30:169-176. 10.1093/mutage/geu045

Chen F, Zhang Y, Senbabaoglu Y, Ciriello G, Yang L, Reznik E, Shuch B, Micevic G, De Velasco G, Shinbrot E, Noble MS, Lu Y, Covington KR, Xi L, Drummond JA, Muzny D, Kang H, Lee J, Tamboli P, Reuter V, Shelley CS, Kaipparettu BA, Bottaro DP, Godwin AK, Gibbs RA, Getz G, Kucherlapati R, Park PJ, Sander C, Henske EP, Zhou JH, Kwiatkowski DJ, Ho TH, Choueiri TK, Hsieh JJ, Akbani R, Mills GB, Hakimi AA, Wheeler DA, and Creighton CJ. 2016. Multilevel Genomics-Based Taxonomy of Renal Cell Carcinoma. Cell Rep 14:2476-2489. 10.1016/j.celrep.2016.02.024 cancer staging manual and the future of TNM. Ann Surg Oncol 17:1471-1474. 10.1245/s10434-0100985-4 
442

443

444

445

446

447

448

449

450

451

452

453

454

455

456

457

458

459

460

461

462

463

464

465

466

467

468

469

470

471

472

in patients with metastatic renal cell carcinoma: PISCES Study. J Clin Oncol 32:1412-1418.

$10.1200 / \mathrm{JCO} .2013 .50 .8267$

Ghidini M, Petrelli F, Ghidini A, Tomasello G, Hahne JC, Passalacqua R, and Barni S. 2017. Clinical development of mTor inhibitors for renal cancer. Expert Opin Investig Drugs 26:1229-1237. $10.1080 / 13543784.2017 .1384813$

Guo H, German P, Bai S, Barnes S, Guo W, Qi X, Lou H, Liang J, Jonasch E, Mills GB, and Ding Z. 2015. The PI3K/AKT Pathway and Renal Cell Carcinoma. J Genet Genomics 42:343-353. 10.1016/j.jgg.2015.03.003

Hager M, Haufe H, Kemmerling R, Hitzl W, Mikuz G, Moser PL, and Kolbitsch C. 2009. Increased activated Akt expression in renal cell carcinomas and prognosis. J Cell Mol Med 13:2181-2188. 10.1111/j.15824934.2008.00488.x

Hager M, Haufe H, Lusuardi L, Schmeller N, and Kolbitsch C. 2011. PTEN, pAKT, and pmTOR expression and subcellular distribution in primary renal cell carcinomas and their metastases. Cancer Invest 29:427-438. 10.3109/07357907.2011.584782

Han G, Zhao W, Song X, Kwok-Shing Ng P, Karam JA, Jonasch E, Mills GB, Zhao Z, Ding Z, and Jia P. 2017. Unique protein expression signatures of survival time in kidney renal clear cell carcinoma through a pan-cancer screening. BMC Genomics 18:678. 10.1186/s12864-017-4026-6

Kim SH, Park WS, Park EY, Park B, Joo J, Joung JY, Seo HK, Lee KH, and Chung J. 2017. The prognostic value of BAP1, PBRM1, pS6, PTEN, TGase2, PD-L1, CA9, PSMA, and Ki-67 tissue markers in localized renal cell carcinoma: A retrospective study of tissue microarrays using immunohistochemistry. PLoS One 12:e0179610. 10.1371/journal.pone.0179610

Kruck S, Bedke J, Hennenlotter J, Ohneseit PA, Kuehs U, Senger E, Sievert KD, and Stenzl A. 2010. Activation of mTOR in renal cell carcinoma is due to increased phosphorylation rather than protein overexpression. Oncol Rep 23:159-163.

Li J, Lu Y, Akbani R, Ju Z, Roebuck PL, Liu W, Yang JY, Broom BM, Verhaak RG, Kane DW, Wakefield C, Weinstein JN, Mills GB, and Liang H. 2013. TCPA: a resource for cancer functional proteomics data. Nat Methods 10:1046-1047. 10.1038/nmeth.2650

Liontos M, Trigka EA, Korkolopoulou P, Tzannis K, Lainakis G, Koutsoukos K, Kostouros E, Lykka M, Papandreou CN, Karavasilis V, Christodoulou C, Papatsoris A, Skolarikos A, Varkarakis I, Adamakis I, Alamanis C, Stravodimos K, Mitropoulos D, Deliveliotis C, Constantinidis CA, Saetta A, Patsouris E, Dimopoulos M, and Bamias A. 2017. Expression and prognostic significance of VEGF and mTOR

Peer) reviewing PDF | (2020:01:45296:2:0:NEW 5 May 2020) 
473

474

475

476

477

478

479

480

481

482

483

484

485

486

487

488

489

490

491

492

493

494

495

496

497

498

499

500

501

502 pathway proteins in metastatic renal cell carcinoma patients: a prognostic immunohistochemical profile for kidney cancer patients. World J Urol 35:411-419. 10.1007/s00345-016-1890-7

Merseburger AS, Hennenlotter J, Kuehs U, Simon P, Kruck S, Koch E, Stenzl A, and Kuczyk MA. 2008. Activation of PI3K is associated with reduced survival in renal cell carcinoma. Urol Int 80:372-377. $10.1159 / 000132694$

Pantuck AJ, Seligson DB, Klatte T, Yu H, Leppert JT, Moore L, O'Toole T, Gibbons J, Belldegrun AS, and Figlin RA. 2007. Prognostic relevance of the mTOR pathway in renal cell carcinoma: implications for molecular patient selection for targeted therapy. Cancer 109:2257-2267. 10.1002/cncr.22677

Que WC, Qiu HQ, Cheng Y, Liu MB, and Wu CY. 2018. PTEN in kidney cancer: A review and meta-analysis. Clin Chim Acta 480:92-98. 10.1016/j.cca.2018.01.031

Rausch S, Schollenberger D, Hennenlotter J, Stuhler V, Kruck S, Stenzl A, and Bedke J. 2019. mTOR and mTOR phosphorylation status in primary and metastatic renal cell carcinoma tissue: differential expression and clinical relevance. J Cancer Res Clin Oncol 145:153-163. 10.1007/s00432-018-2775-5

Rini BI, Campbell SC, and Escudier B. 2009. Renal cell carcinoma. Lancet 373:1119-1132. 10.1016/S01406736(09)60229-4

Siegel RL, Miller KD, and Jemal A. 2020. Cancer statistics, 2020. CA Cancer J Clin 70:7-30. $10.3322 /$ caac. 21590

Sui J, Yang S, Liu T, Wu W, Xu S, Yin L, Pu Y, Zhang X, Zhang Y, Shen B, and Liang G. 2019. Molecular characterization of lung adenocarcinoma: A potential four-long noncoding RNA prognostic signature. J Cell Biochem 120:705-714. 10.1002/jcb.27428

Tang L, Li X, Gao Y, Chen L, Gu L, Chen J, Lyu X, Zhang Y, and Zhang X. 2017. Phosphatase and tensin homolog (PTEN) expression on oncologic outcome in renal cell carcinoma: A systematic review and meta-analysis. PLoS One 12:e179437. 10.1371/journal.pone.0179437

Tang Z, Kang B, Li C, Chen T, and Zhang Z. 2019. GEPIA2: an enhanced web server for large-scale expression profiling and interactive analysis. Nucleic Acids Res 47:W556-W560. 10.1093/nar/gkz430

Zhang Y, Kwok-Shing Ng P, Kucherlapati M, Chen F, Liu Y, Tsang YH, de Velasco G, Jeong KJ, Akbani R, Hadjipanayis A, Pantazi A, Bristow CA, Lee E, Mahadeshwar HS, Tang J, Zhang J, Yang L, Seth S, Lee S, Ren X, Song X, Sun H, Seidman J, Luquette LJ, Xi R, Chin L, Protopopov A, Westbrook TF, Shelley CS, Choueiri TK, Ittmann M, Van Waes C, Weinstein JN, Liang H, Henske EP, Godwin AK, Park PJ, Kucherlapati R, Scott KL, Mills GB, Kwiatkowski DJ, and Creighton CJ. 2017. A Pan- 
503

504

505

506

507

508

509

510

511

512

513

514

515

516

517
Cancer Proteogenomic Atlas of PI3K/AKT/mTOR Pathway Alterations. Cancer Cell 31:820-832 e823. 10.1016/j.ccell.2017.04.013

Zhao Z, Azadzoi KM, Choi HP, Jing R, Lu X, Li C, Wang F, Lu J, and Yang JH. 2017. LC-MS/MS Analysis Unravels Deep Oxidation of Manganese Superoxide Dismutase in Kidney Cancer. Int J Mol Sci 18. 10.3390/ijms18020319

Zhao Z, Liu Y, Liu Q, Wu F, Liu X, Qu H, Yuan Y, Ge J, Xu Y, and Wang H. 2019. The mRNA Expression Signature and Prognostic Analysis of Multiple Fatty Acid Metabolic Enzymes in Clear Cell Renal Cell Carcinoma. J Cancer 10:6599-6607. 10.7150/jca.33024

Zhao Z, Lu J, Qu H, Wang Z, Liu Q, Yang X, Liu S, Ge J, Xu Y, Li N, and Yuan Y. 2018. Evaluation and prognostic significance of manganese superoxide dismutase in clear cell renal cell carcinoma. Hum Pathol 80:87-93. 10.1016/j.humpath.2017.12.032

Zhu C, Wei J, Tian X, Li Y, and Li X. 2015. Prognostic role of PPAR-gamma and PTEN in the renal cell carcinoma. Int J Clin Exp Pathol 8:12668-12677.

Zhu L, Wang Y, He J, Tang J, Lv W, and Hu J. 2018. Cytoplasmic SQSTM1/ P62 Accumulation Predicates a Poor Prognosis in Patients with Malignant Tumor. J Cancer 9:4072-4086. 10.7150/jca.26399 
Figure 1

A schematic diagram to investigate the expression and prognostic profiling of $\mathrm{PI3K/AKT/mTOR} \mathrm{signaling} \mathrm{pathway} \mathrm{members} \mathrm{in} \mathrm{ccRCC.}$

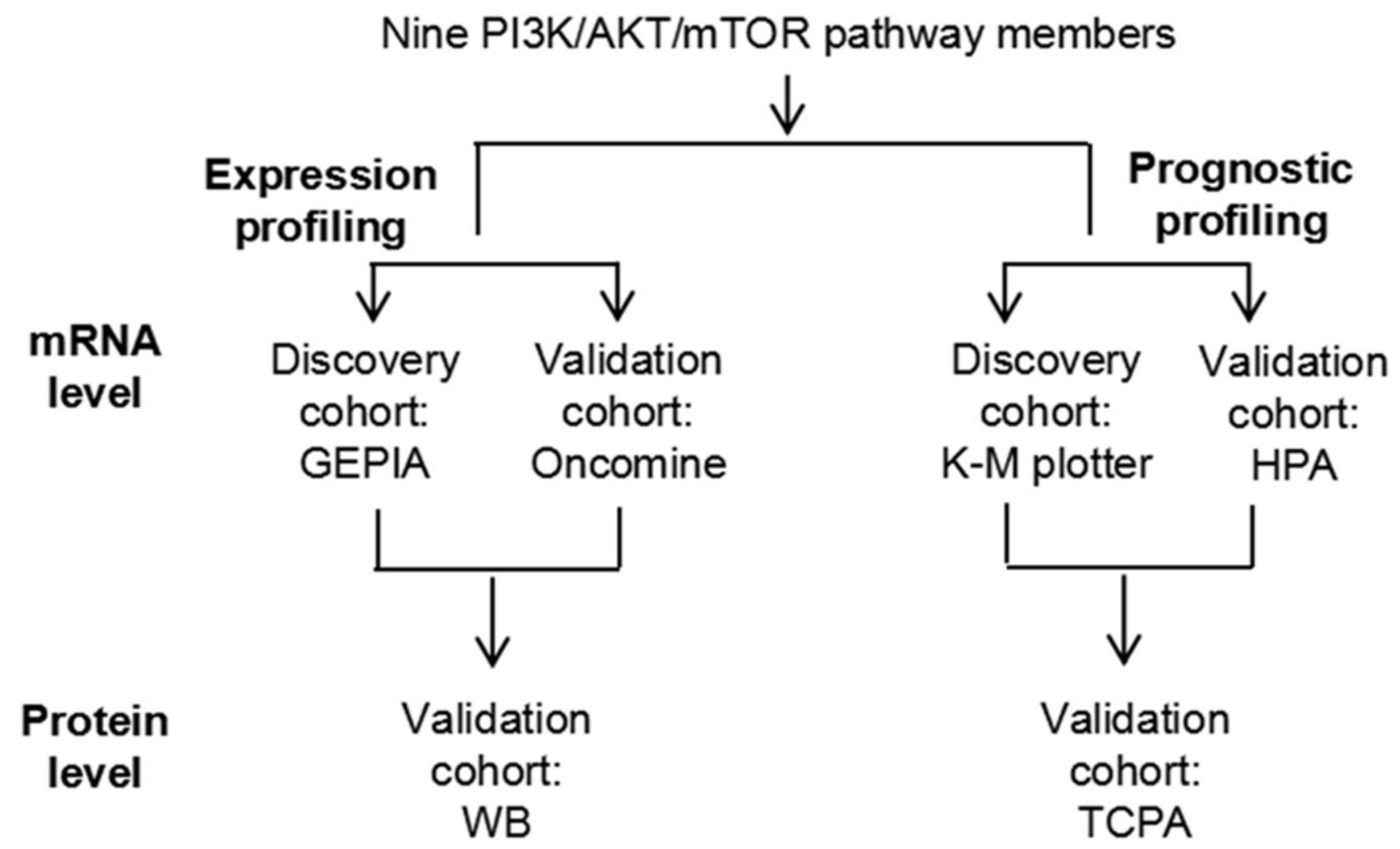




\section{Figure 2}

GEPIA mRNA expression profiles of PI3K/AKT/mTOR signaling pathway proteins in 523 cCRCC (T) and 100 normal ones (N), which was downloaded from TCGA and GTEx databases (http://gepia2.cancer-pku.cn/).

Box plots demonstrate the relative mRNA expression levels $(\log 2(T P M+1))$ of (A) PTEN, (B) PIK3CA, (C) PIK3CB, (D) PIK3CD, (E) PIK3CG, (F) AKT1, (G) AKT2, (H) AKT3 and (I) mTOR, which were not significantly altered between the tissues $(P>0.05)$. 

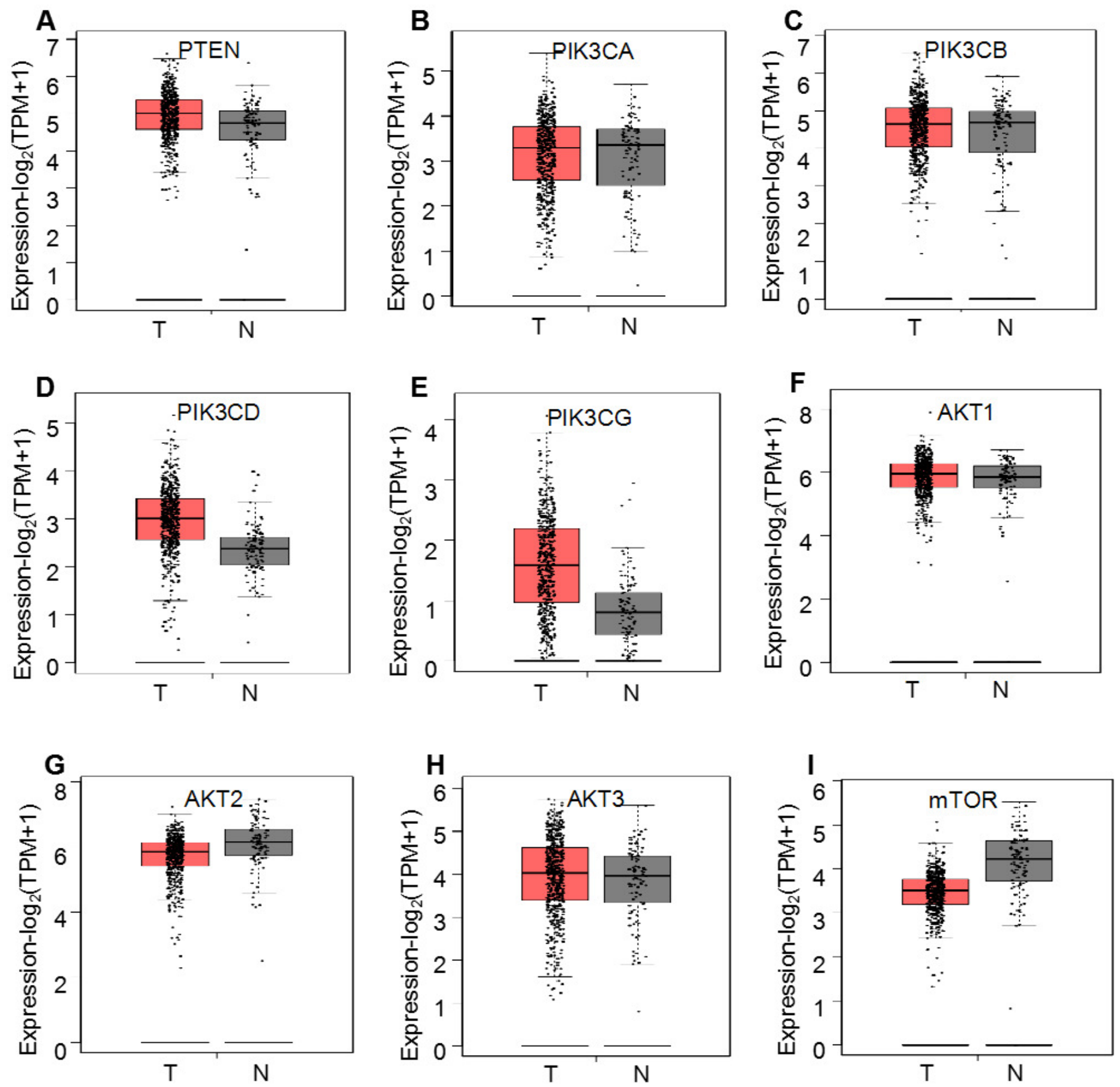


\section{Figure 3}

GEPIA mRNA expression profiles of PI3K/AKT/mTOR pathway members in 523 cCRCC tissues, which investigated the association of their mRNA expression with pathological stages of ccRCC patients.

Violin plots demonstrate the relationship between mRNA expression levels $(\log 2(T P M+1))$ of (A) PTEN, (B) PIK3CA, (C) PIK3CB, (D) PIK3CD, (E) PIK3CG, (F) AKT1, (G) AKT2, (H) AKT3 and (I) $m T O R$, which were not significantly altered between the tissues $(P>0.05)$. 

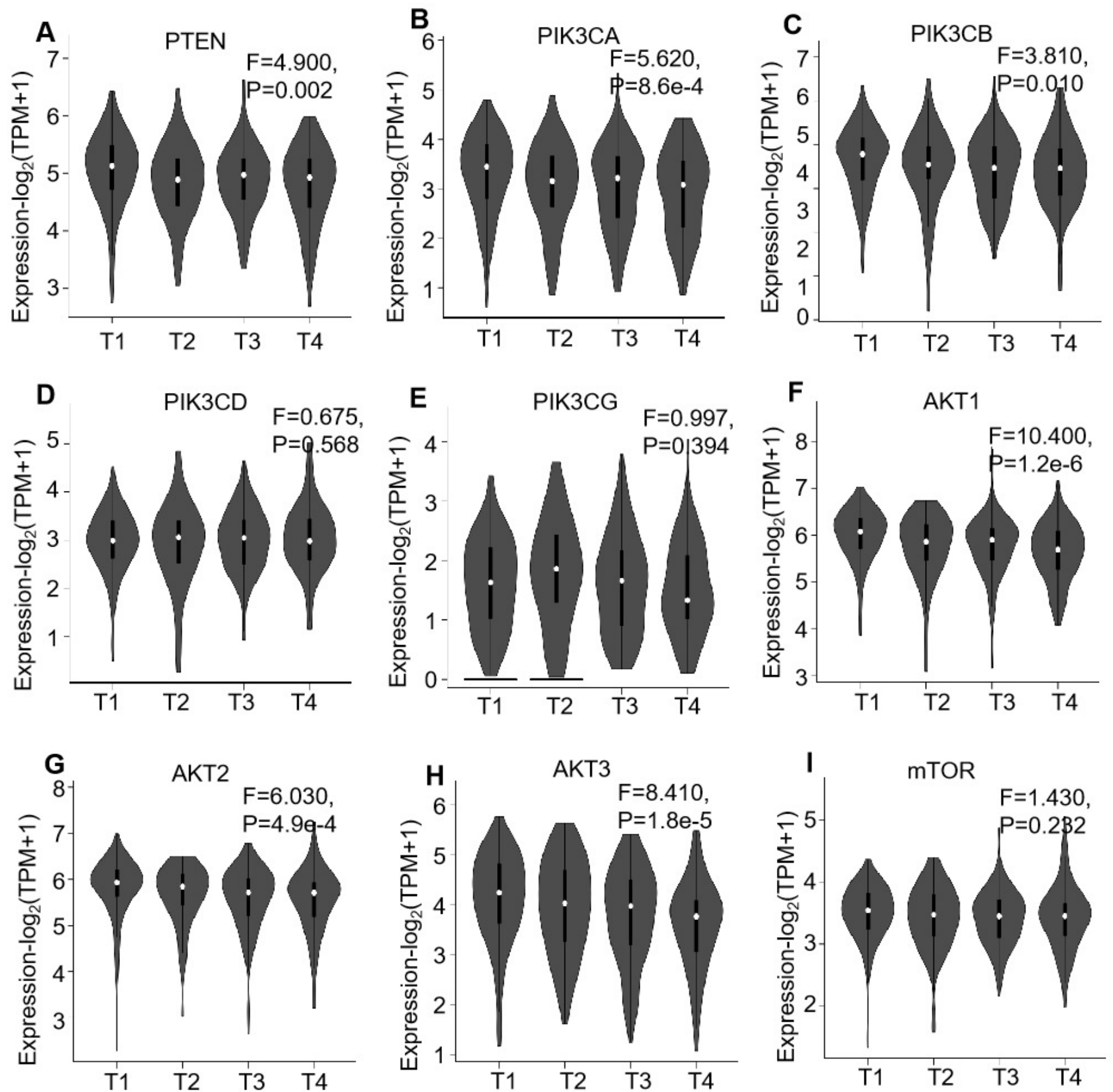


\section{Figure 4}

Validation of PI3K, AKT and mTOR protein expression in cCRCC by WB.

(A) WB for PI3K, AKT and mTOR expression of three individual ccRCC (T) and adjacent normal tissues (N). Actin as a loading control (left). (B) The statistic results of gray value ratios of the WB bands in CCRCC and normal tissues (right).

$\begin{array}{lllllll}\text { A } & \mathrm{N} 1 & \mathrm{~T} 1 & \mathrm{~N} 2 & \mathrm{~T} 2 & \mathrm{~N} 3 & \mathrm{~T} 3\end{array}$

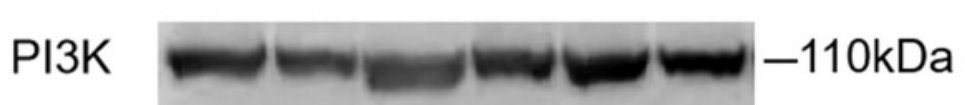

Akt mTOR

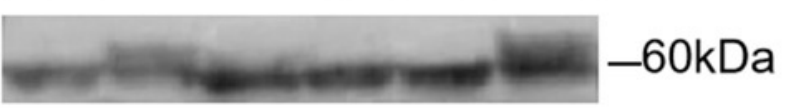
Actin
B

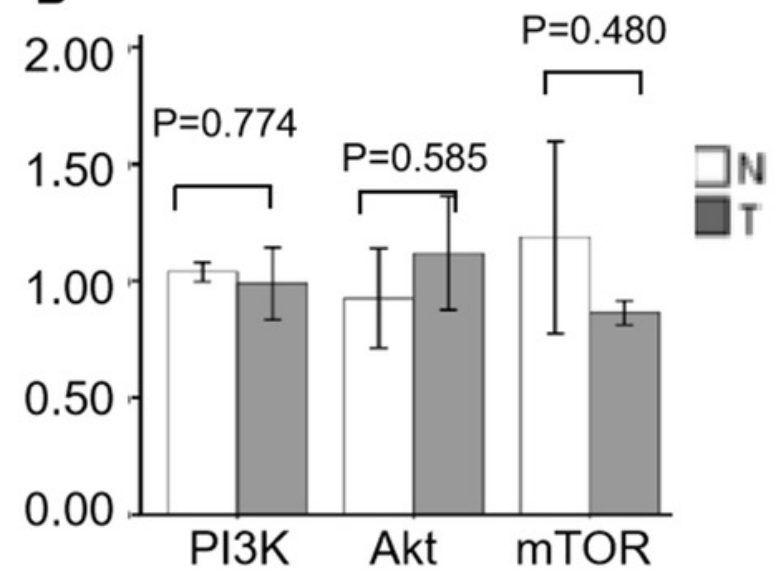


Figure 5

K-M plotter mRNA analysis displaying the overall survival curves of PI3K/AKT/mTOR signaling pathway members.

The overall survival curves of (A) PTEN, (B) PIK3CA, (C) PIK3CB, (D) PIK3CD, (E) PIK3CG, (F) AKT1, (G) AKT2, (H) AKT3 and (I) mTOR expression levels in 530 patients with clear cell renal cell carcinoma. 

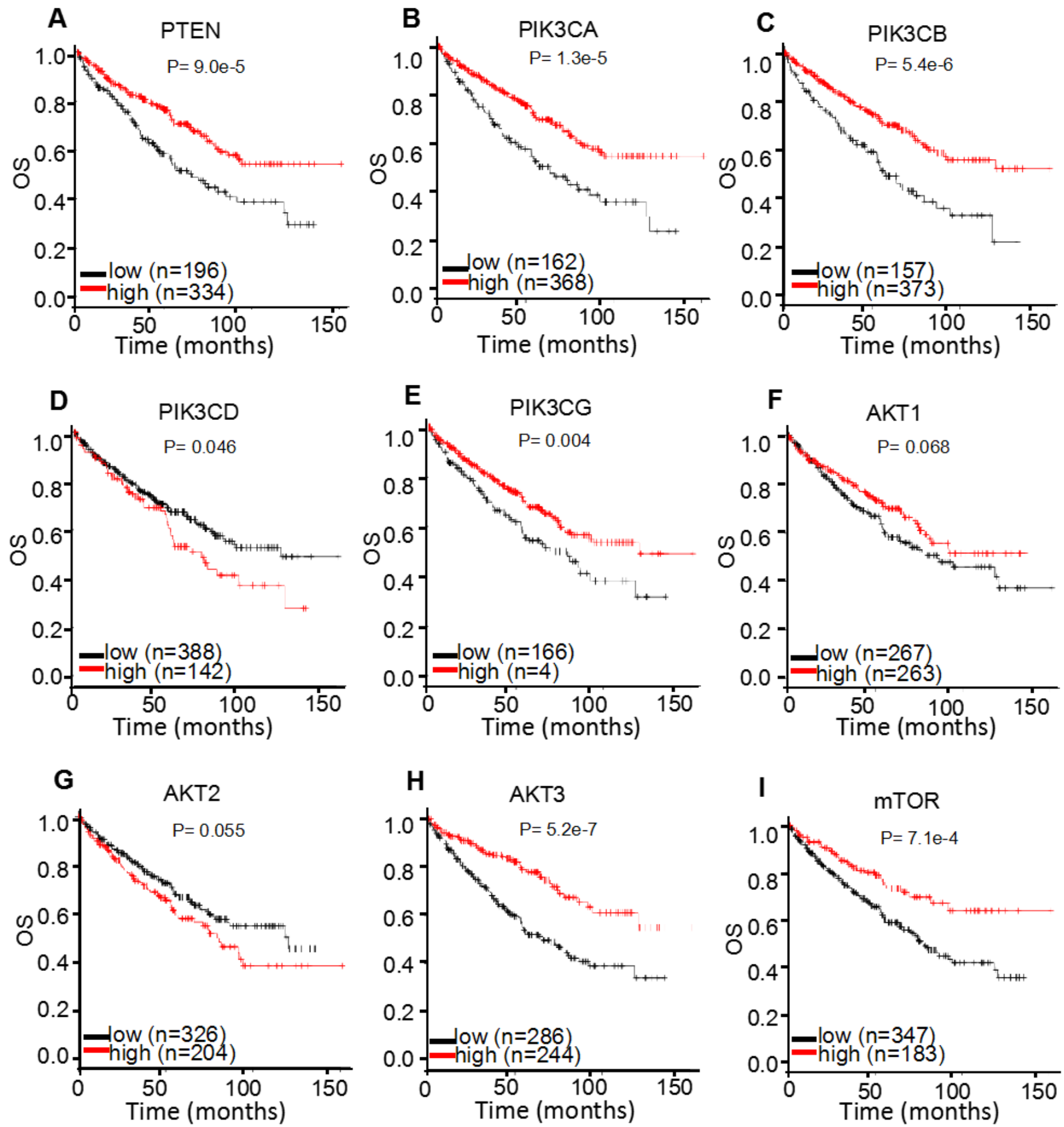
Figure 6

HPA mRNA expression analysis displaying the overall survival curves of PI3K/AKT/mTOR signaling pathway members.

The overall survival curves of (A) PTEN, (B) PIK3CA, (C) PIK3CB, (D) PIK3CD, (E) PIK3CG, (F) AKT1, (G) AKT2, (H) AKT3 and (I) mTOR in 528 patients with clear cell renal cell carcinoma.
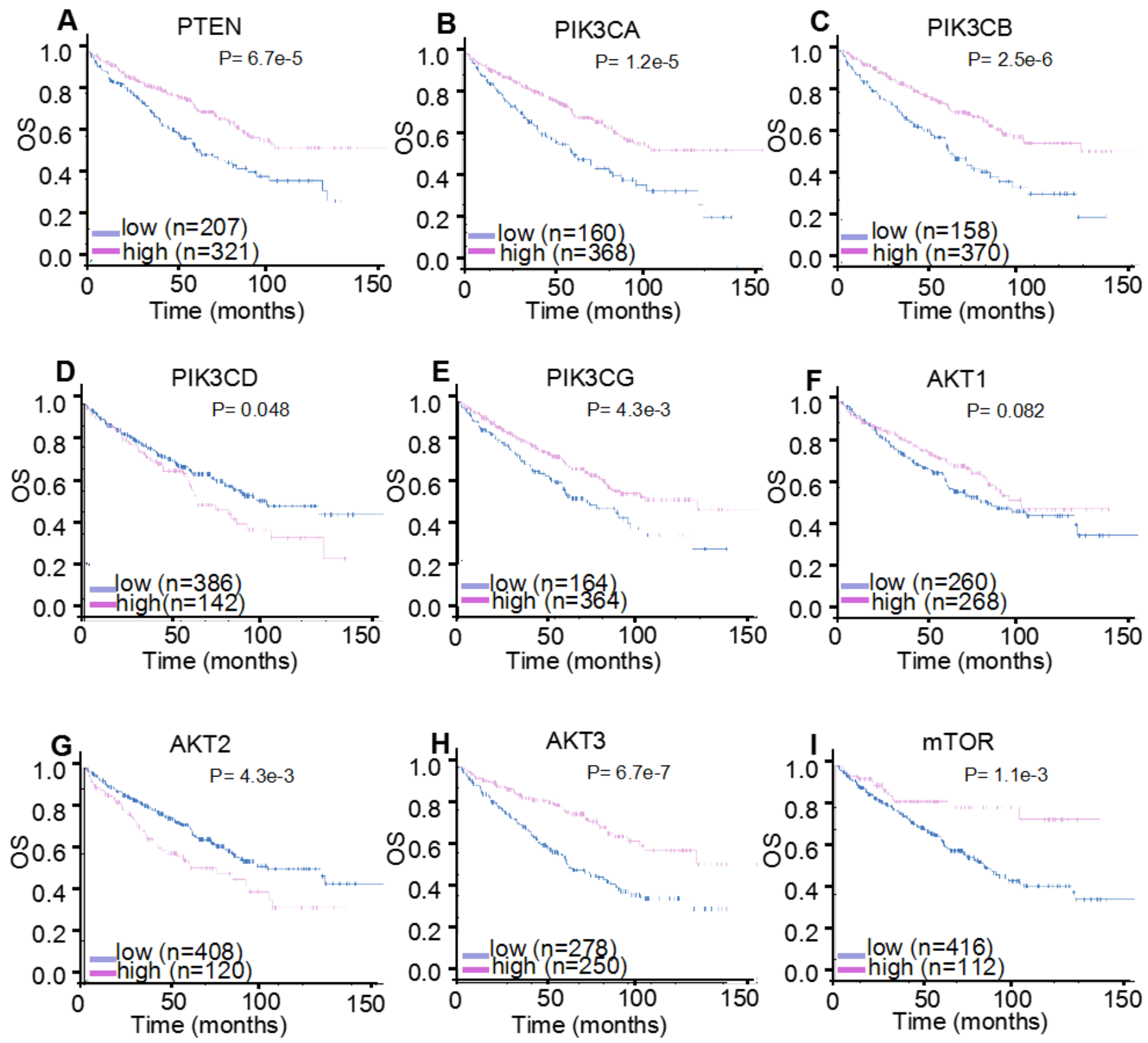


\section{Figure 7}

TCPA protein expression analysis displaying the overall survival curves of $\mathrm{PISK} / \mathrm{AKT} / \mathrm{mTOR}$ signaling pathway members.

The overall survival curves of (A) PTEN, (B) PIK3CA, (C) AKT, (D) p-AKT (T308), (E) p-AKT (S473), (F) mTOR and (G) p-mTOR (S2448) in 445 patients with clear cell renal cell carcinoma. 

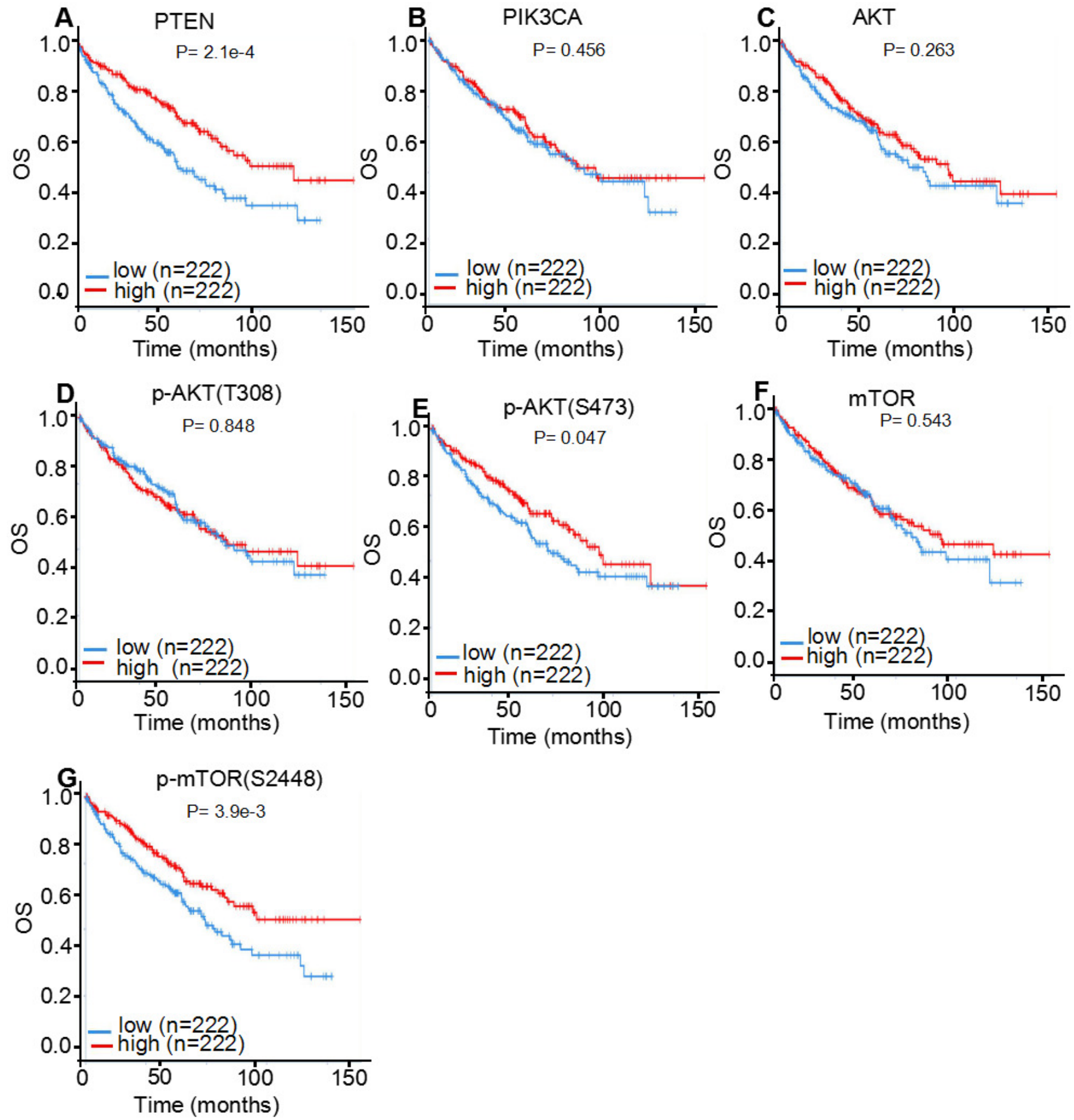
Table $\mathbf{1}$ (on next page)

Correlation of the PI3K/AKT/mTOR mRNA expression with OS in cCRCC patients (K-M plotter, $n=530$ ). 
1 Table 1. Correlation of the PI3K/AKT/mTOR mRNA expression with OS in ccRCC

2 patients (K-M plotter, $\mathbf{n}=\mathbf{5 3 0})$.

3

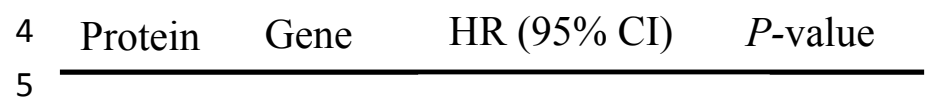

6 PTEN PTEN $0.56(0.41-0.75)<0.001$

7 PI3K PIK3CA $0.52(0.38-0.70)<0.001$

8

9

10

11

12

13

14

15

16

PIK3CB $\quad 0.50(0.37-0.68)<0.001$

PIK3CD $1.38(1.01-1.91) \quad 0.046$

PIK3CG $0.64(0.47-0.87) \quad 0.004$

AKT $\quad$ AKT1 $\quad 0.76(0.56-1.02) \quad 0.068$

AKT2 $1.34(0.99-1.82) \quad 0.055$

AKT3 $\quad 0.44(0.32-0.62)<0.001$

$\begin{array}{llll}\text { mTOR } & \text { mTOR } & 0.54(0.38-0.78) & <0.001\end{array}$ 


\section{Table 2 (on next page)}

Correlation of the PI3K/AKT/mTOR mRNA expression with OS in different clinical stage of ccRCC patients (K-M plotter, $n=530$ ). 
1 Table 2. Correlation of the PI3K/AKT/mTOR mRNA expression with OS in different

2 clinical stage of ccRCC patients $(\mathrm{K}-\mathrm{M}$ plotter, $\mathrm{n}=\mathbf{5 3 0})$.

\begin{tabular}{|c|c|c|c|c|}
\hline$=$ Gene & Stage & $\mathrm{n}^{1}$ & HR $(95 \% \mathrm{CI})$ & $P$-value \\
\hline \multirow{4}{*}{ PTEN } & $\mathrm{I}$ & 265 & $0.46(0.25-0.85)$ & 0.011 \\
\hline & II & 57 & $3.28(1.04-10.37)$ & 0.034 \\
\hline & III & 123 & $0.44(0.25-0.79)$ & 0.005 \\
\hline & IV & 82 & $0.51(0.30-0.87)$ & 0.012 \\
\hline \multirow{4}{*}{ PIK3CA } & $\mathrm{I}$ & 265 & $0.48(0.26-0.88)$ & 0.016 \\
\hline & II & 57 & $2.08(0.67-6.48)$ & 0.200 \\
\hline & III & 123 & $0.51(0.29-0.89)$ & 0.017 \\
\hline & IV & 82 & $0.61(0.37-1.00)$ & 0.049 \\
\hline \multirow{4}{*}{ PIK3CB } & $\mathrm{I}$ & 265 & $0.43(0.23-0.81)$ & 0.007 \\
\hline & II & 57 & $2.14(0.47-9.73)$ & 0.310 \\
\hline & III & 123 & $0.40(0.22-0.70)$ & 0.001 \\
\hline & IV & 82 & $0.41(0.25-0.67)$ & $<0.001$ \\
\hline \multirow{4}{*}{ PIK3CD } & $\mathrm{I}$ & 265 & $1.41(0.74-2.70)$ & 0.300 \\
\hline & II & 57 & $2.25(0.69-7.33)$ & 0.170 \\
\hline & III & 123 & $0.55(0.31-0.96)$ & 0.033 \\
\hline & $\mathrm{IV}$ & 82 & $2.42(1.25-4.68)$ & 0.007 \\
\hline \multirow{4}{*}{ PIK3CG } & I & 265 & $0.53(0.28-1.00)$ & 0.045 \\
\hline & II & 57 & $0.29(0.08-1.00)$ & 0.046 \\
\hline & III & 123 & $0.31(0.17-0.54)$ & $<0.001$ \\
\hline & IV & 82 & $0.59(0.34-1.03)$ & 0.062 \\
\hline \multirow{4}{*}{ AKT1 } & $\mathrm{I}$ & 265 & $0.44(0.24-0.80)$ & 0.006 \\
\hline & II & 57 & $3.00(0.98-9.20)$ & 0.044 \\
\hline & III & 123 & $2.15(1.14-4.07)$ & 0.015 \\
\hline & IV & 82 & $1.28(0.78-2.10)$ & 0.330 \\
\hline \multirow[t]{4}{*}{ AKT2 } & I & 265 & 1. $65(0.91-2.99)$ & 0.093 \\
\hline & II & 57 & $2.22(0.76-7.14)$ & 0.130 \\
\hline & III & 123 & $1.58(0.90-2.78)$ & 0.110 \\
\hline & IV & 82 & $1.61(0.96-2.68)$ & 0.067 \\
\hline \multirow{4}{*}{ AKT3 } & $\mathrm{I}$ & 265 & $0.56(0.31-1.02)$ & 0.054 \\
\hline & II & 57 & $0.43(0.09-1.95)$ & 0.260 \\
\hline & III & 123 & $0.45(0.25-0.81)$ & 0.006 \\
\hline & IV & 82 & $0.50(0.27-0.94)$ & 0.029 \\
\hline \multirow{4}{*}{ mTOR } & I & 265 & $0.30(0.12-0.77)$ & 0.008 \\
\hline & II & 57 & $0.24(0.06-0.86)$ & 0.018 \\
\hline & III & 123 & $0.58(0.33-1.02)$ & 0.053 \\
\hline & IV & 82 & $1.36(0.82-2.26)$ & 0.240 \\
\hline
\end{tabular}


$4 1 \longdiv { }$

42 Note: ${ }^{1}$ The total number was 527 , because there were missing expression values and/ or 43 incomplete survival data. 


\section{Table 3 (on next page)}

Correlation of the PI3K/AKT/mTOR mRNA expression with OS in different sex of ccRCC patients ( $K-M$ plotter, $n=530$ ). 
1 Table 3. Correlation of the PI3K/AKT/mTOR mRNA expression with OS in different sex

2 of ccRCC patients (K-M plotter, $n=530)$.

\begin{tabular}{|c|c|c|c|c|}
\hline Gene & Sex & $\mathrm{n}$ & $\operatorname{HR}(95 \% \mathrm{CI})$ & $P$-value \\
\hline \multirow[t]{2}{*}{ PTEN } & female & 186 & $0.35(0.21-0.59)$ & $<0.001$ \\
\hline & male & 344 & $0.53(0.36-0.77)$ & $<0.001$ \\
\hline \multirow[t]{2}{*}{ PIK3CA } & female & 186 & $0.27(0.15-0.51)$ & $<0.001$ \\
\hline & male & 344 & $0.57(0.39-0.82)$ & 0.003 \\
\hline \multirow[t]{2}{*}{ PIK3CB } & female & 186 & $0.45(0.27-0.76)$ & 0.002 \\
\hline & male & 344 & $0.48(0.33-0.70)$ & $<0.001$ \\
\hline \multirow[t]{2}{*}{ PIK3CD } & female & 186 & $0.57(0.34-0.97)$ & 0.037 \\
\hline & male & 344 & $1.81(1.22-2.68)$ & 0.003 \\
\hline \multirow[t]{2}{*}{ PIK3CG } & female & 186 & $0.49(0.30-0.82)$ & 0.006 \\
\hline & male & 344 & $0.68(0.47-0.99)$ & 0.044 \\
\hline \multirow[t]{2}{*}{ AKT1 } & female & 186 & $0.51(0.31-0.85)$ & 0.008 \\
\hline & male & 344 & $0.76(0.49-1.17)$ & 0.210 \\
\hline \multirow[t]{2}{*}{ AKT2 } & female & 186 & $0.76(0.46-1.25)$ & 0.270 \\
\hline & male & 344 & $1.71(1.15-2.55)$ & 0.007 \\
\hline \multirow[t]{2}{*}{ AKT3 } & female & 186 & $0.32(0.20-0.54)$ & $<0.001$ \\
\hline & male & 344 & $0.48(0.31-0.72)$ & $<0.001$ \\
\hline \multirow[t]{2}{*}{ mTOR } & female & 186 & $0.27(0.14-0.52)$ & $<0.001$ \\
\hline & male & 344 & $0.78(0.54-1.14)$ & 0.200 \\
\hline
\end{tabular}


Table 4 (on next page)

Correlation of the PTEN/PI3K/AKT/mTOR protein expression with OS in cCRCC patients (TCPA, $n=445$ ). 
1 Table 4. Correlation of the PTEN/PI3K/AKT/mTOR protein expression with OS in ccRCC

2 patients (TCPA, $n=445)$.

3

4

5

7 9 10

11

12

13

14

15

16 Note: ${ }^{1}$ Abbreviation for univariate $\operatorname{Cox} P$.

17

18

19 